\title{
DATA ASSIMILATION EXPERIMENTS USING QUALITY CONTROLLED AIRS VERSION 5 TEMPERATURE SOUNDINGS
}

\author{
Joel Susskind and Oreste Reale \\ NASA GSFC and GEST/UMBC
}

\begin{abstract}
The AIRS Science Team Version 5 retrieval algorithm has been finalized and is now operational at the Goddard DAAC in the processing (and reprocessing) of all AIRS data. The AIRS Science Team Version 5 retrieval algorithm contains two significant improvements over Version 4: 1) Improved physics allows for use of AIRS observations in the entire 4.3 $\mu \mathrm{m} \mathrm{CO}_{2}$ absorption band in the retrieval of temperature profile $T(p)$ during both day and night. Tropospheric sounding $15 \mu \mathrm{m} \mathrm{CO} \mathrm{CO}_{2}$ observations are now used primarily in the generation of cloud cleared radiances $\hat{R}_{i}$. This approach allows for the generation of accurate values of $\hat{R}_{i}$ and $T(p)$ under most cloud conditions. 2) Another very significant improvement in Version 5 is the ability to generate accurate case-by-case, level-by-level error estimates for the atmospheric temperature profile, as well as for channel-bychannel error estimates for $\hat{\mathrm{R}}_{\mathrm{i}}$. These error estimates are used for quality control of the retrieved products.

We have conducted forecast impact experiments assimilating AIRS temperature profiles with different levels of quality control using the NASA GEOS-5 data assimilation system. Assimilation of quality controlled $T(p)$ resulted in significantly improved forecast skill compared to that obtained from analyses obtained when all data used operationally by NCEP, except for AIRS data, is assimilated. We also conducted an experiment assimilating AIRS radiances uncontaminated by clouds, as done operationally by ECMWF and NCEP. Forecast resulting from assimilated AIRS radiances were of poorer quality than those obtained assimilating AIRS temperatures.
\end{abstract}

Index Terms-Data Assimilation, NWP, AIRS/AMSU, Quality Control, Temperature Profile

\section{AIRS VERSION 5 TEMPERATURE PROFILE QC}

AIRS Version 5 retrievals contain case-by-case level-bylevel error estimates for all accepted profiles. These error estimates are used to determine a case-by-case characteristic pressure $p_{\text {good }}$, down to which the profile is considered acceptable. All accepted profiles are assigned to have high quality down to at least $70 \mathrm{mb}$. The characteristic pressure $\mathrm{p}_{\text {good }}$ is defined as the highest pressure (somewhere between $70 \mathrm{mb}$ and $\mathrm{p}_{\text {surf }}$ ) at which the error is not greater than a pressure dependent error estimate threshold. These pressure dependent thresholds were optimized in Version 5 bearing in mind what was considered to be the best trade-off between accuracy and spatial coverage for use in both dataassimilation and climate applications.

Figure 1a shows the rms error of global quality controlled temperature profiles for Version 4 (black) and Version 5 (gray) using the standard Version 5 thresholds. Figure 1b shows the percent of cases accepted for both Version 4 and for Version 5 using the standard cutoffs. The global percent accepted cases for Version 5 Standard is significantly higher than that of Version 4 at $300 \mathrm{mb}$ with a comparable accuracy. The same is true at all levels of the troposphere. The red and purple curves represent quality controlled Version 5 retrievals using tighter cutoffs as shown in the figure. They show how tightening thresholds can lead to significantly more accurate quality controlled retrievals, but with a lower percentage of accepted retrievals, resulting in poorer spatial coverage.

Figure 2 shows the spatial distribution of quality controlled temperatures at $700 \mathrm{mb}$. Areas with surface pressure less than $700 \mathrm{mb}$, such as over East-Antarctica, show up as data gaps in this figure. The Version 4 spatial coverage over land and extratropical oceans at $700 \mathrm{mb}$ is extremely low. Version 5 Standard has extensive $700 \mathrm{mb}$ spatial coverage globally. Version 5 Tight has spatial coverage at $700 \mathrm{mb}$ closer to that of Version 4, and Version 5 Medium has intermediate spatial coverage. All Version 5 coverages are extensive over ocean $50^{\circ} \mathrm{N}-50^{\circ} \mathrm{S}$.

\section{FORECAST IMPACT EXPERIMENTS}

We have conducted a number of data assimilation experiments as a step toward finding an optimum balance of spatial coverage and sounding accuracy with regard to improving forecast skill. The data assimilation and forecast system used is the GEOS-5 DAS, which represents a combination of the NASA GEOS-5 forecast model with the NCEP operational Grid Point Statistical Interpolation (GSI) global analysis scheme. All analyses and forecasts were run at a $0.5^{\circ} \times 0.5^{\circ}$ spatial resolution.

A number of experiments utilizing AIRS data were conducted. In all experiments, data was assimilated for the period January 1- January 31, 2003. Seven day forecasts were run every two days beginning January 6,2003 , and 
forecasts every 12 hours were verified against the NCEP analysis, which was taken as "truth".

The first set of experiments assimilated quality controlled AIRS Version 5 retrieved temperatures down to the surface using the three different quality control thresholds described in Section 1. The AIRS Version 5 temperature profiles were presented to the GSI analysis as rawinsonde profiles.

Forecasts generated from these three sets of analyses are compared to those from the "Control" analysis, in which all the data used operationally by NCEP was assimilated, but no AIRS data was assimilated. Radiances from the Aqua AMSU A instrument were also assimilated operationally by NCEP and are included in the "Control". It should be noted that the Aqua orbit (1:30 ascending) is almost identical to that of NOAA 16 carrying HIRS3, AMSU A and AMSU $B$, so AIRS/AMSU temperature soundings are providing additional information to that contained in the AMSU A/AMSU B radiances on NOAA 16 in the same orbit, as well as those of the Aqua AMSU A radiances. No AIRS data was assimilated operationally at that time.

Figure 3 shows the average of the 2712 hour to seven day forecast sea level pressure (SLP) anomaly correlation coefficients verified against the NCEP analysis for both Northern Hemisphere extra-tropics and Southern Hemisphere extra-tropics for all these experiments. In the Northern Hemisphere, assimilating AIRS soundings resulted in an improvement in average five day forecast skill of roughly five hours for all three sets of AIRS assimilations. At seven days, the Tight assimilation maintained this improvement in skill, while the improvement dropped off slightly with loosening the QC thresholds. In the Southern Hemisphere extra-tropics, the same relative forecast skill, resulting from the three AIRS assimilations, held. This time, however, the Tight assimilation maintained control forecast skill at 7 days, while addition of more sounding data actually degraded forecast skill as judged by SLP. Results shown for this set of experiments indicate that assimilation of Quality Controlled AIRS temperature soundings extending down to the surface significantly improves forecast skill in the Northern Hemisphere extratropics. Assimilation of soundings with Tight Quality Control performed better than with Medium or Standard Quality Control in both hemispheres.

NCEP and ECMWF now assimilate AIRS observations operationally. The current operational practice is to directly assimilate observed AIRS radiances rather than AIRS temperature soundings as done in the first set of experiments. The operational methodologies used by both NCEP and ECMWF do not have the capability to derive and assimilate cloud cleared AIRS radiances. Instead, the analysis procedures used at both Centers select and assimilate only these AIRS observations which are "thought to be unaffected by clouds." These uncontaminated radiance observations are influenced primarily from temperatures in the stratosphere and also above clouds in areas where clouds are present. Our results from AIRS indicate that roughly
$95 \%$ of AIRS pixels are cloud contaminated. Therefore, information from most tropospheric sounding AIRS observations is not included in the operational AIRS radiance assimilation process.

We conducted a second set of experiments, which were designed to assess the contribution of assimilation of AIRS tropospheric temperatures derived under primarily partially cloudy conditions toward the improved forecast skill. In the first of these experiments, we used the AIRS Tight Quality Control, but only assimilated those soundings down to at most $200 \mathrm{mb}$. In the second new experiment, we assimilated AIRS radiances according to the NCEP operational procedure. In the AIRS Radiance Assimilation experiments, all other data assimilated in the Control was also included, but no AIRS temperature profile data was assimilated.

Figure 4 shows analogous results to those shown in Figure 3, but for Northern Hemisphere and Southern Hemisphere Extra-Tropics $500 \mathrm{mb}$ Geopotential Height $\left(\mathrm{GPH}_{500}\right)$ anomaly coefficients. Results are shown for forecasts using the control (black) and AIRS Version 5 Tight (red) analyses as before. Also shown are results from the AIRS Version 5 Tight down to $200 \mathrm{mb}$ (orange) and AIRS Radiance Assimilation Analyses (green). Figure 4a shows the very significant result that virtually all the positive impact of assimilation of Quality Controlled AIRS temperature soundings in the Northern Hemisphere ExtraTropics is lost if only AIRS stratospheric temperatures are used in the assimilation process (orange). The most important information is coming from tropospheric temperatures which are determined and assimilated primarily in partially cloudy scenes. In the Southern Hemisphere Extra-Tropics, the Tight QC assimilation improved 7 day forecast skill, as judged by the $\mathrm{GPH}_{500}$ anomaly correlation. This improvement was lost when only stratospheric soundings were assimilated.

Assimilation of AIRS radiances unaffected by clouds results in a substantially reduced forecast impact in the Northern Hemisphere Extra-Tropics, and a significant negative forecast impact in the Southern Hemisphere ExtraTropics, compared to the assimilation of AIRS Tight Quality Controlled temperature profiles down to the surface. At least a part of this reduction in forecast impact of radiance assimilation in the Northern Hemisphere ExtraTropics, results from the significant loss of spatial coverage of AIRS tropospheric sounding channels used in the data assimilation process due to cloud contamination. The cause of the substantial negative impact in the Southern Hemisphere Extra-Tropics is unclear.

It is apparent that assimilation of quality controlled AIRS temperature profiles performs better than assimilation of AIRS observed radiances. We are conducting further studies to optimize the best trade between spatial coverage and accuracy for NWP purposes. We will also assess the assimilation of quality controlled values of AIRS clear column radiances $\hat{R}_{j}$ for NWP purposes. 
LAYER MEAN RMS TEMPERATURE $\left({ }^{\circ} \mathrm{C}\right)$

GLOBAL DIFFERENCES FROM ECMWF January 25,2003

Global

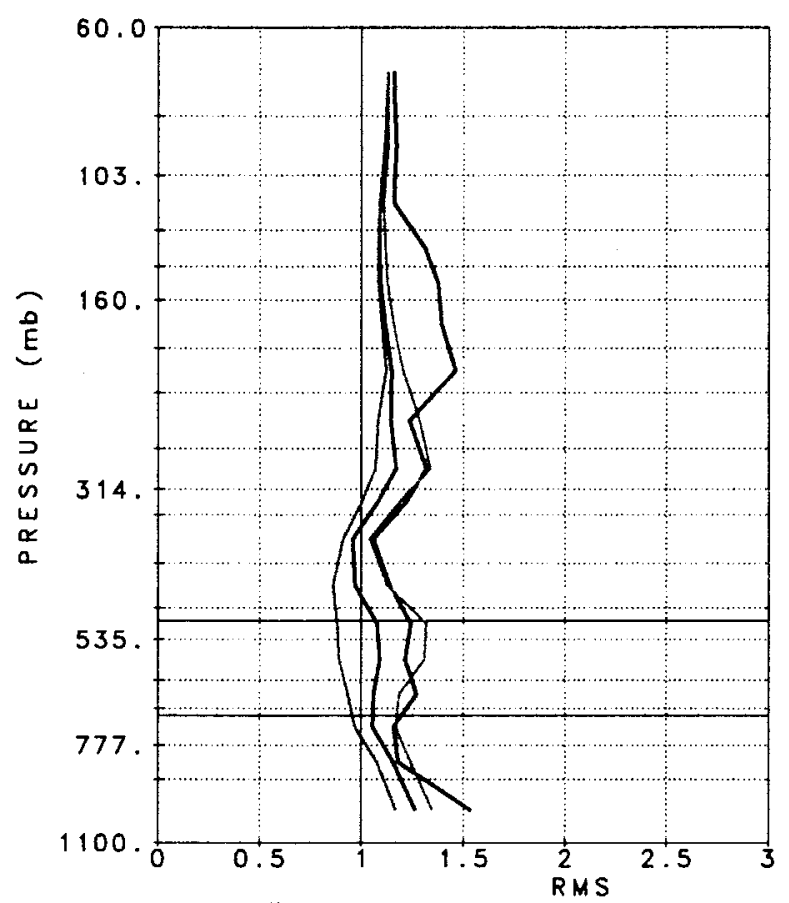

—us standord

Ws Modium
Figure 1a
Porcont of IR/MW Cases Included

Jonuory 25, 2003

Global

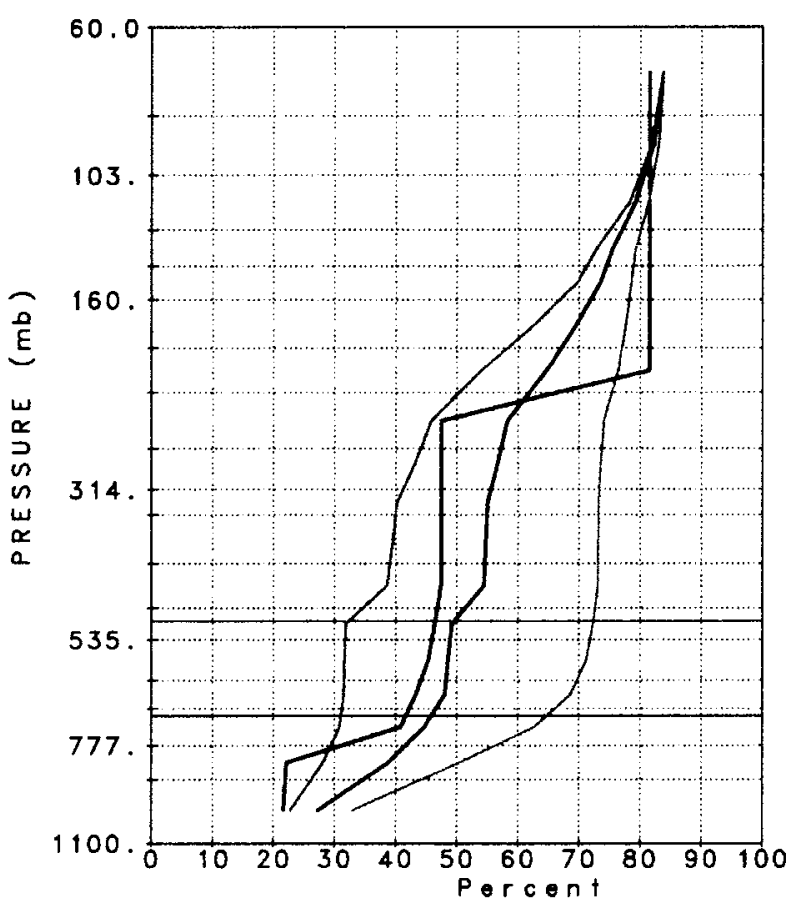

vs stondard

Zus vedtum
Figure 1b
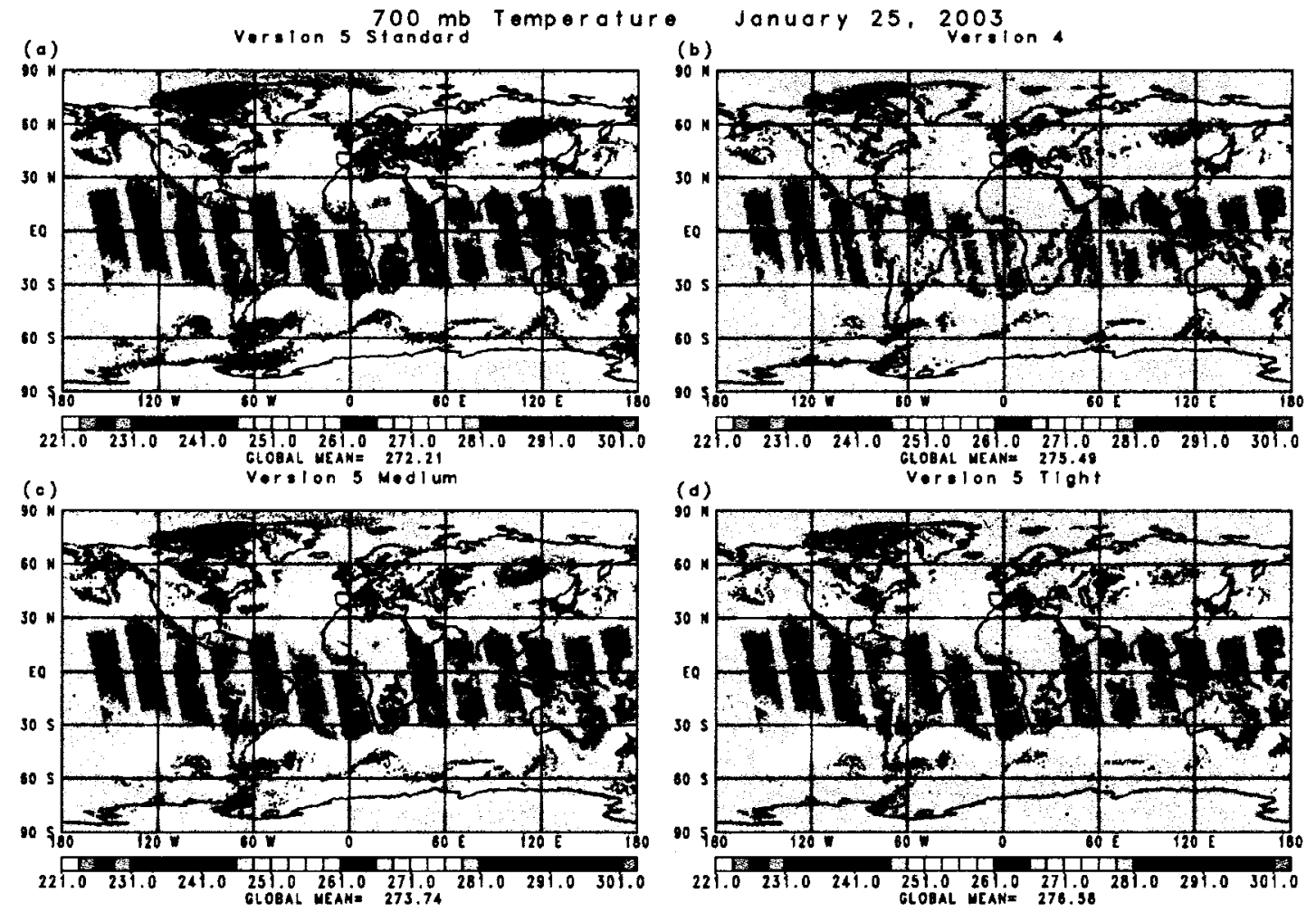

Figure 2 


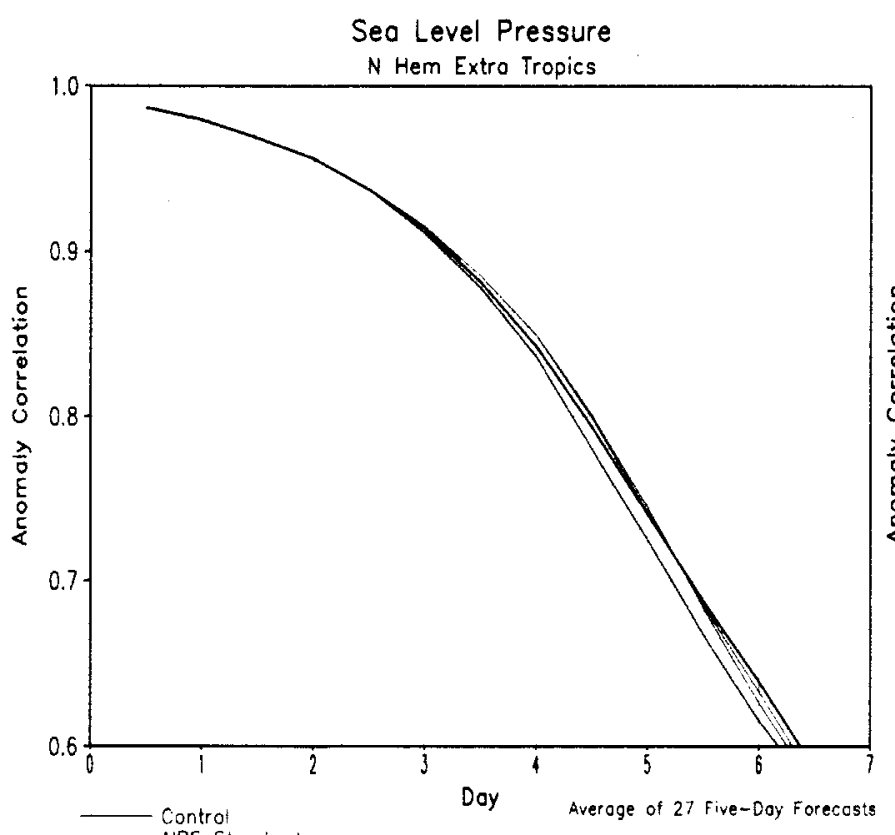

—_ AIRS Standord -...... AIRS Medium AIRS Tight

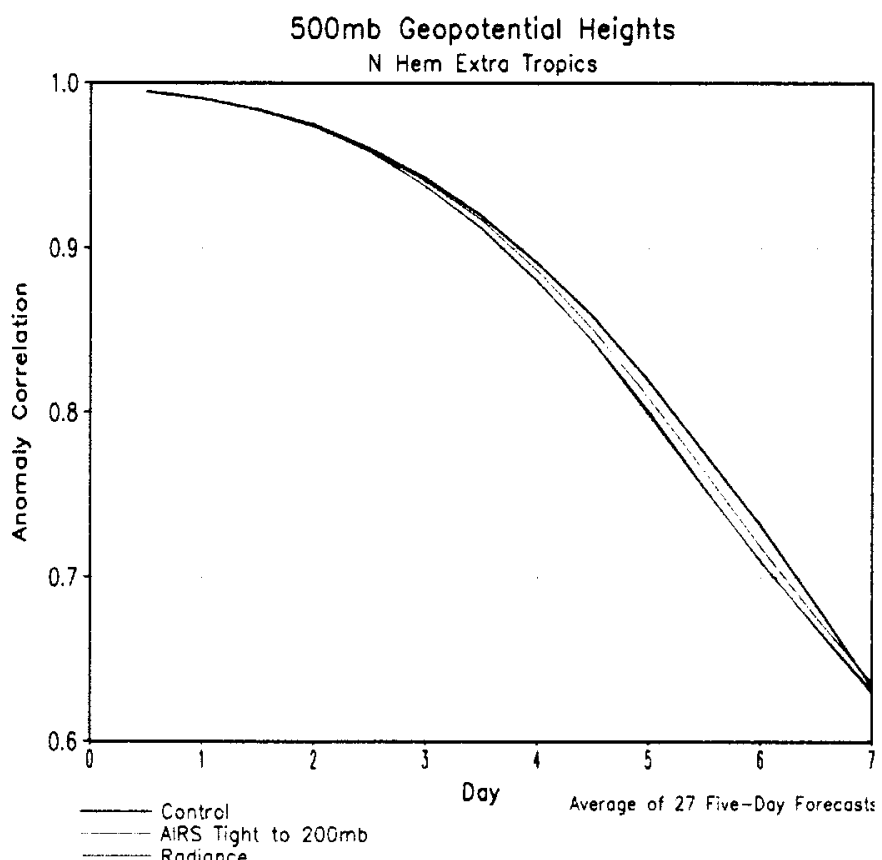

Figure $4 \mathrm{a}$

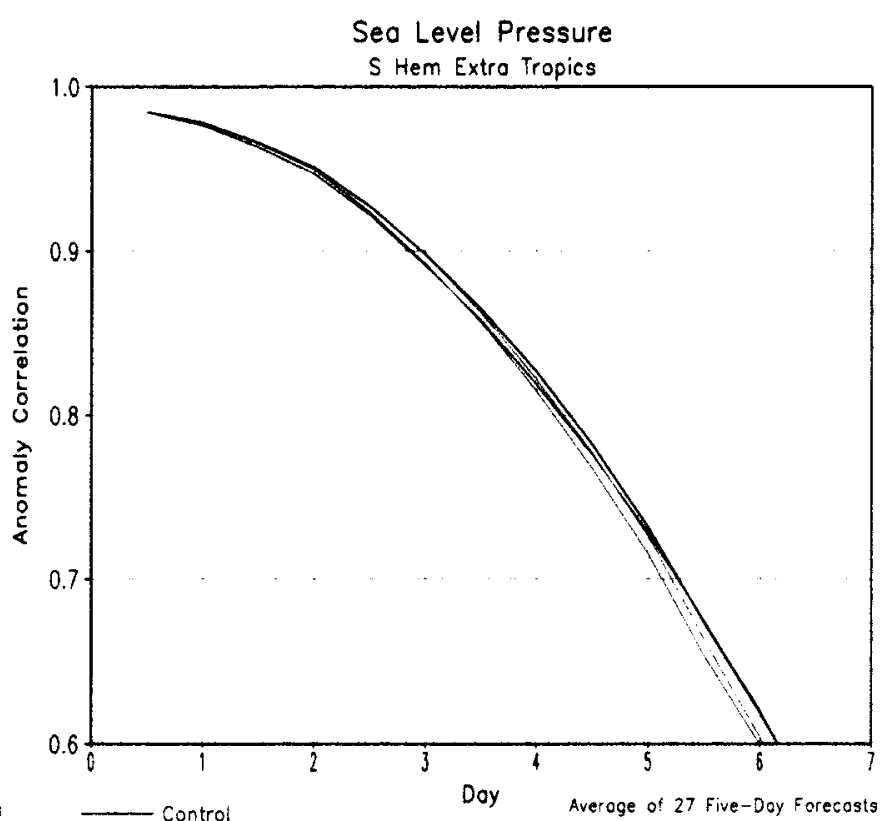

Figure $3 a$
…... AIRS Standard AIRS Medium
Figure $3 b$

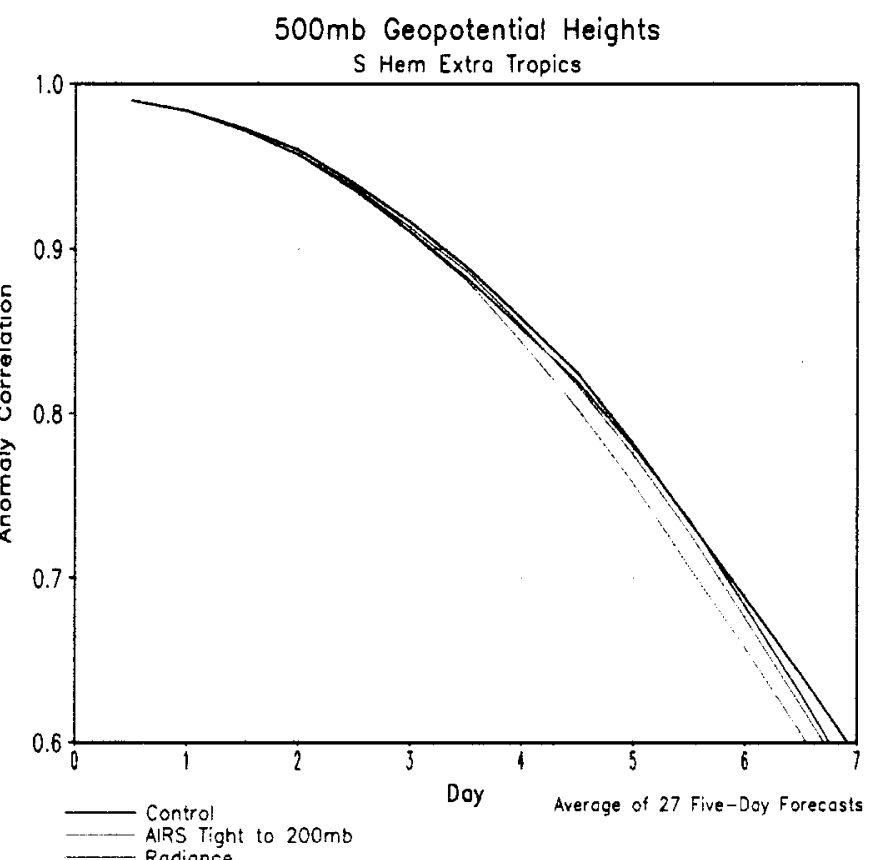

Figure $4 b$ 